\title{
NORMAL SPACES WHOSE STONE-ČECH REMAINDERS HAVE COUNTABLE TIGHTNESS
}

\author{
JIN-YUAN ZHOU
}

(Communicated by Franklin D. Tall)

\begin{abstract}
We prove, assuming PFA, that each normal space whose StoneČech remainder has countable tightness is ACRIN. A normal space $X$ is called ACRIN if each of its regular images is normal. Fleissner and Levy proved that if $X$ is normal and every countably compact subset of the Stone-Čech remainder $\beta X \backslash X$ is closed in $\beta X \backslash X$, then $X$ is ACRIN. They asked if each normal space whose Stone-Čech remainder has countable tightness is ACRIN. Theorem 2 gives the positive answer assuming the Proper Forcing Axiom.
\end{abstract}

It is well known that the tightness of $\beta \omega \backslash \omega$ is $2^{\omega}$. Since every not countably compact Hausdorff space contains a closed copy of $\omega$, the next lemma is easy to prove.

Lemma 1. If $X$ is a normal space and $\beta X \backslash X$ has countable tightness, then $X$ is countably compact.

Theorem 2 (PFA). If $X$ is a normal space and $\beta X \backslash X$ has countable tightness, then $X$ is $A C R I N$.

Proof. Let $f: X \rightarrow Y$ be a continuous map and $Y$ be regular. We prove that $Y$ is normal. By virtue of Lemma 5 of [FL] there exist $Z$ and $b f$ such that $X \subseteq Z \subseteq \beta X$ and $b f$ is a perfect map from $Z$ onto $Y$ with $\left.b f\right|_{X}=f$. Since $\beta X \backslash X$ has countable tightness, it is easy to see that the spaces $X, Y$, and $Z$ are all countably compact. Since perfect mappings preserve normality, we only need to prove that $Z$ is normal. Let $K$ and $L$ be two disjoint closed subsets of $Z$. We will prove that $\bar{K}^{\beta X} \cap \bar{L}^{\beta X}=\varnothing$. Since $X$ is normal, we have $\overline{K \cap X}^{\beta X} \cap \overline{L \cap X}^{\beta X}=\varnothing$. Take open subsets $U$ and $V$ of $\beta X$ such that $\bar{U}^{\beta X} \cap \bar{V}^{\beta X}=\varnothing, \overline{K \cap X}^{\beta X} \subseteq U$, and $\overline{L \cap X}^{\beta X} \subseteq V$. Let $U^{\prime}=U \backslash \bar{L}^{\beta x}$, $V^{\prime}=V \backslash \bar{K}^{\beta X}$, and $T=\left(\overline{K \cup L}^{\beta \bar{X}}\right) \backslash\left(U^{\prime} \cup V^{\prime}\right)$. Obviously $T$ is a closed subset of $\beta X$ and is contained in $\beta X \backslash X$. Thus $T$ is a compact space of countable tightness. Furthermore, $K \backslash U$ and $L \backslash V$ are contained in $T$ and are closed subsets of the countably compact space $Z$. We have proved that $K \backslash U$ and $L \backslash V$ are countably compact subsets of a compact space of countable tightness. By virtue of Balogh's Theorem [Ba, 2.1], $K \backslash U$ and $L \backslash V$ are compact. Thus

Received by the editors November 23, 1990 and, in revised form, July 18, 1991.

1991 Mathematics Subject Classification. Primary 54C05, 54D15, 54D40. 
we have

$$
\begin{aligned}
\bar{K}^{\beta X} \cap \bar{L}^{\beta X} & =\left({\overline{K \cap U^{\beta X}}}^{\beta} \cup(K \backslash U)\right) \cap\left(\overline{L \cap V}^{\beta X} \cup(L \backslash V)\right) \\
& =\left({\overline{K \cap U^{\beta X}}}^{\beta X}(L \backslash V)\right) \cup\left(\overline{L \cap V}^{\beta X} \cap(K \backslash U)\right) \\
& \subseteq\left(\bar{K}^{\beta X} \cap L\right) \cup\left(\bar{L}^{\beta X} \cap K\right)=\varnothing .
\end{aligned}
$$

We are done.

\section{ACKNOWLEDGMENT}

We acknowledge the referee's help in writing this paper.

\section{REFERENCES}

[Ba] Z. Balogh, On compact Hausdorff spaces of countable tightness, Proc. Amer. Math. Soc. 105 (1989), 755-764.

[FL] W. Fleissner and R. Levy, Stone-Čech remainders which make continuous images normal, Proc. Amer. Math. Soc. 106 (1988), 839-842.

Department of Mathematics, Sichuan University, Chengdu, Sichuan 610064, People's REPUBLIC OF CHINA

Current address: Department of Mathematic and Statistics, York University, 4700 Keele Street, North York, Ontario, Canada M3J 1 P3

E-mail address: ysma9202@clid.yorku.ca 